\title{
DETERMINACIÓN DE LA FLORA POLINÍFERA DEL VALLE DE OXAPAMPA (PASCO-PERÚ) EN BASE A ESTUDIOS PALINOLÓGICOS
}

\section{DETERMINATION OF POLLEN FLORA OF OXAPAMPA VALLEY (PASCO- PERU) BASED ON PALYNOLOGICAL STUDIES}

\author{
Rosario Sayas Rivera ${ }^{1}$ y Luis Huamán Mesía²
}

\begin{abstract}
Resumen
Con el fin de evaluar la flora polinífera del valle de Oxapampa, se evaluaron 19 muestras de polen corbicular obtenidos de 7 apiarios de los distritos de Huancabamba, Chontabamba y Oxapampa. Se realizó un inventario florístico para obtener un herbario y una palinoteca de referencia, las muestras de polen corbicular se obtuvieron a través de trampas caza-polen. Las muestras de polen corbicular fueron separadas en grupos de acuerdo al color de los acúmulos de polen, realizándose cálculos de frecuencia. Las muestras de flora de referencia y polen corbicular fueron acetolizadas y luego analizadas por microscopia para la identificación de los tipos polínicos o taxa, correlacionándose el color de los acúmulos de polen corbicular con las especies identificadas en las muestras.

Los análisis palinológicos dieron como resultado la presencia de 47 taxa distribuídos en 27 familias botánicas, siendo Dictyocaryum lamarckianum, Juglans neotropica y Cyrtocymura scorpioides, las especies que alcanzaron las mayores frecuencias relativas en todo el Valle de Oxapampa. Los colores más frecuente del polen corbicular fueron el marrón, amarillo y el naranja, con una variedad de colores en las especies Vernonanthura patens, Cecropia sp, Cyrtocymura scorpioides y Baccharis latifolia.
\end{abstract}

Palabras clave: Polen corbicular, Flora polinífera, Oxapampa, Apis mellifera L.

\begin{abstract}
In order to evaluate the potential of pollen flora of Oxapampa Valley (Pasco-Perú), 19 samples of bee pollen were evaluated, collected from 7 apiaries on the districts of Huancabamba, Chontabamba and Oxapampa. We conducted a floristic inventory for a herbarium and a reference pollen library. Corbicula pollen samples were obtained through hunting-pollen traps. Corbicula pollen samples were separated into groups according to the color of the pollen accumulation and estimates of specific pollen frequency were performed by these groups of color. The reference samples of flora and Corbicula pollen were acetolized and analyzed by microscopy to identify the morphological types of taxa present, correlating the color of the Corbicula pollen accumulation with species identified in samples.

Palynological analysis performed on samples of bee pollen resulted in the presence of 47 taxa distributed in 27 botanical families, Dictyocaryum lamarckianum, Juglans neotropica and Cyrtocymura scorpioides were the species that reached the highest relative frequencies across Oxapampa Valley. The most frequent colors of the Corbicula pollen were brown, yellow and orange. The Corbicula pollen of the species Vernonanthura patens, Cecropia sp, Cyrtocymura scorpioides and Baccharis latifolia showed a greater variety of shades of colors.
\end{abstract}

Key words: Corbicular pollen, Pollen flora, Oxapampa, Apis mellifera L.

\section{Introducción.}

El polen corbicular es un acúmulo de polen colectado por Apis mellifera L. que es humedecido con néctar y miel para transportarlo a la colmena en las corbículas de sus patas posteriores (Sáenz \& Gómez, 2000; Sá-Otero et al., 2002; Ramírez \& Montenegro, 2004), este producto, en general, posee alto contenido de proteínas, carbohidratos, cenizas, fibra y bajo contenido de lípidos (Lazcano et al. 2006).

La flora que utiliza la abeja melífera como fuente de polen se denomina flora polinífera, siendo su identificación importante para incrementar el desarrollo de la actividad apícola. La producción del polen corbicular cuenta con gran demanda por parte de los distribuidores de productos naturales.

Para determinar la relación entre las abejas y esta flora se realizan estudios palinológicos, basados en el reconocimiento de los tipos morfológicos del polen presente en los acúmulos, así el estudio de las cargas polínicas a través del análisis microscópico ha sido la herramienta más utilizada por diversos autores para poder identificar su procedencia botánica (Sempe et al., 1989; Sá-Otero et al., 2002; Salamanca et al., 
2002; Faye et al., 2002; Montenegro \& Pizarro, 2006).

Tabla 1. Ubicación de los puntos de muestreo.

\begin{tabular}{|c|c|c|c|c|}
\hline Estudios palinológicos & Distrito & Apiario & Coordenadas (UTM) & $\begin{array}{l}\text { Altitud } \\
\text { (msnm) }\end{array}$ \\
\hline realizado en diversos & Huancabamba & Illimo & 8849630 N y 18445005 E & 1950 \\
\hline dous a palses & & 2 de mayo & 8829191 N y 18451376 E & 1985 \\
\hline latinoamericanos & Chontabamba & San Marcos & 8825968 N y $18455201 \mathrm{E}$ & 2010 \\
\hline (Salamanca et al., 2002, Girón, 1995), & & La Florida & 8828981 N y 18455232 E & 1825 \\
\hline Brasil (Da Silva et al., 2006; Hatsue, & & Cantarizú & 8819273 N y $18457601 \mathrm{E}$ & 1695 \\
\hline 2006; Hatsue et al., 2007; Da Luz et al., & Oxapampa & San Alberto & 8831320 N y 18456686 E & 1828 \\
\hline 2007; Carpes, 2008); Chile (Sempe & & Acuzazú & 8838598 N y 18453866 E & 1829 \\
\hline
\end{tabular}
al. 1989; Montenegro et al., 1992; Ramírez \& Montenegro, 2004; Contreras, 2004); y Argentina (Faye et al., 2002; Fegúndez \& Caccavara, 2003; Faye \& Molinelli, 2004; Baldi et al., 2004; Fegúndez et al., 2006). En el Perú se han realizado diversos estudios sobre morfología polínica (Isayama, 1987-1988; Ventura \& Huamán, 2008), determinación de flora apícola (Zevallos \& Higaona, 1988), potencial melífero (Zevallos \& Pérez, 1990) y análisis de procedencia de la miel de abeja (León et al., 19891990) de diferentes zonas del país, con estos resultados se han llegado a plantear calendarios apícolas como el de Zevallos (1986).

La presente investigación caracterizó la flora polinífera del valle de Oxapampa, región que posee una importante producción apícola en el Perú, esta caracterización, además es importante para plantear estrategias de conservación de las diferentes especies poliníferas de la región, muchas de las cuales son especies nativas.

adicionalmente, se colectaron flores en antesis de cada planta las que sirvieron para obtener los tipos polínicos referenciales. Las plantas fueron secadas e identificadas en la estación del Missouri Botanical Garden en Oxapampa; y depositadas en los Herbarios Selva Central (HOXA) y en el Herbario de la Universidad Peruana Cayetano Heredia (HUPCH).

Las muestras de polen corbicular se obtuvieron mediante trampas caza-polen ubicadas en las piqueras de las colmenas colocadas entre las 9:00 am y las 11:00 am, por ser estas las horas de mayor actividad de las abejas. De cada apiario se eligieron 3 colmenas al azar, con excepción del distrito de Huancabamba, donde se muestreo un apiario, y en él una colmena. Las muestras colectadas fueron colocadas en frascos de plástico, rotuladas y secadas cuidadosamente. Las muestras de polen corbicular fueron transportados al Laboratorio de Palinología y Paleobotánica de la Universidad Peruana Cayetano Heredia (LPP-UPCH) para su procesamiento y análisis microscópico.

\section{Materiales y métodos.}

Zonas de muestreo y Obtención de muestras.

El valle de Oxapampa está ubicado a 12 horas de Lima en la margen derecha del río Chontabamba en la provincia de Oxapampa del departamento de Pasco (Figura 1), evaluándose 19 muestras de polen corbicular, de esta zona, durante el mes de agosto del 2007, de 7 apiarios ubicados en los distritos de Huancabamba,

Chontabamba y Oxapampa. La ubicación de los puntos de muestreo se detalla en la Tabla 1.

Inventario florístico y obtención de muestras de polen corbicular

Se colectaron especies vegetales en floración ubicadas a 700 metros alrededor de cada uno de los apiarios incluidos en la presente investigación, para su determinación botánica;

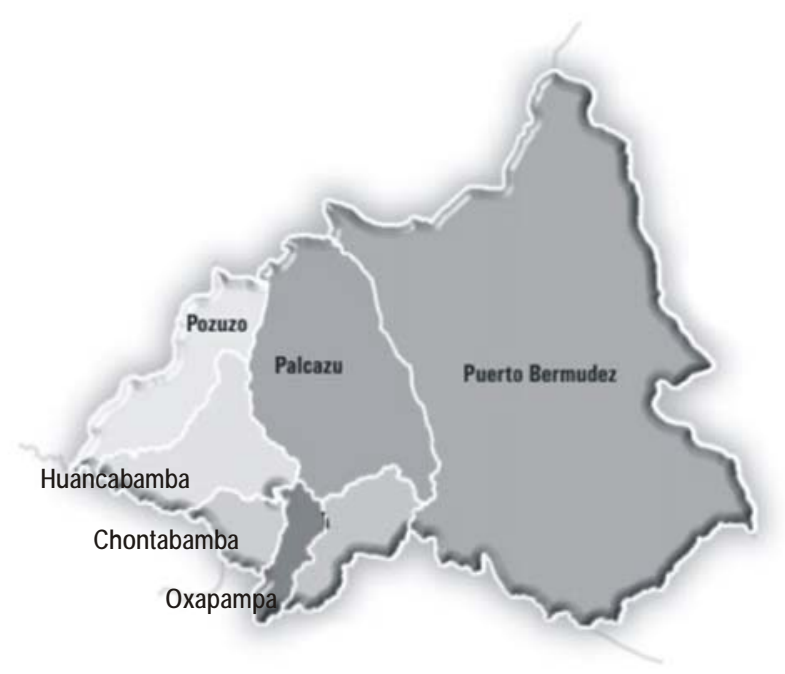

Figura 1. Número de hormigas (Formicidae) contabilizadas mensualmente (mayo 2007 a abril 2008) en 21 viviendas de la ciudad de Puerto Vallarta, Jalisco, México. Triángulos oscuros: con inclusión de Solenopsis sp. en el conteo. Cuadros claros: sin inclusión de Solenopsis sp. en el conteo.

Análisis palinológicos y elaboración de la palinoteca de referencia

Las muestras de flores fueron procesadas por el 
método de acetólisis (Erdtman, 1966), realizándose, luego, el análisis microscópico obteniéndose los tipos polínicos de referencia, los cuales fueron almacenados en la Palinoteca del Laboratorio de Palinología y Paleobotánica de la Universidad Peruana Cayetano
Heredia (LPP-UPCH). Estas muestras fueron confrontadas con sus respectivas excicatas determinadas y depositadas en el HUPCH.

Las muestras de polen corbicular fueron pesadas y cuidadosamente separadas por colores, siguiendo el

Tabla 2. Contenido porcentual de los taxones identificados en los tres distritos muestreados del Valle de Oxapampa.

\begin{tabular}{|c|c|c|c|c|c|}
\hline \multirow{2}{*}{ Familia } & \multirow{2}{*}{ Especie } & \multicolumn{3}{|c|}{ Porcentaje por distritos } & \multirow{2}{*}{ Porcentaje } \\
\hline & & Huancabamba & Chontabamba & Oxapampa & \\
\hline Arecaceae & Dictyocaryum lamarckianum (Mart.)H. Wendl & & $37.27 \%$ & $24.14 \%$ & $32.18 \%$ \\
\hline Juglandaceae & Juglans neotropica Diles & & $28.51 \%$ & $10.65 \%$ & $21.18 \%$ \\
\hline Asteraceae & Cyrtocymura scorpioides (Lam.) H. Rob & & $13.89 \%$ & $6.10 \%$ & $10.45 \%$ \\
\hline Asteraceae & Vernonanthura patens (Kunth) H. Rob. & & & $20.66 \%$ & $9.09 \%$ \\
\hline Asteraceae & Baccharis latifolia Ruiz \& Pav. & & $0.31 \%$ & $14.95 \%$ & $6.63 \%$ \\
\hline Euphorbiaceae & Croton perpeciosus Croizat & $87.16 \%$ & $0.01 \%$ & $2.99 \%$ & $5.11 \%$ \\
\hline Poaceae & Zea mays L. & & $0.64 \%$ & $11.41 \%$ & $4.97 \%$ \\
\hline Desconocido & Desconocido IV & & $5.83 \%$ & & $3.37 \%$ \\
\hline Solanaceae & Solanaceae Tipo II & $5.13 \%$ & $0.86 \%$ & $0.78 \%$ & $1.04 \%$ \\
\hline Asteraceae & Munnozia hastifolia (Poepp.) H. Rob. \&Brettell & & & $2.07 \%$ & $0.91 \%$ \\
\hline Cucurbitaceae & Cucurbita sp & & $0.89 \%$ & $1.76 \%$ & $0.86 \%$ \\
\hline Malvaceae & Sida rhomboidea Roxb. ex Fleming & & $0.53 \%$ & $1.65 \%$ & $0.73 \%$ \\
\hline Cecropiaceae & Cecropia sp & $7.18 \%$ & $0.35 \%$ & $0.19 \%$ & $0.49 \%$ \\
\hline Pinnaceae & Pinus tecunumanii F. Schwerdtf. ex Eguiluz \& J.P.Perry & & $0.72 \%$ & & $0.41 \%$ \\
\hline Solanaceae & Acnistus arborescens (L.) Schltdl. & & $0.62 \%$ & & $0.36 \%$ \\
\hline Passifloraceae & Passiflora ligularis Juss. & & $0.55 \%$ & & $0.32 \%$ \\
\hline Asteraceae & Trixis divaricata (Kunth) Spreng. & & $6.22 \%$ & $0.13 \%$ & $0.18 \%$ \\
\hline Asteraceae & Ageratina sp & & $0.12 \%$ & $0.20 \%$ & $0.16 \%$ \\
\hline Fabaceae & Inga sp & & $0.27 \%$ & & $0.16 \%$ \\
\hline Solanaceae & Solanaceae Tipo I & & $0.22 \%$ & & $0.13 \%$ \\
\hline Anacardiaceae & Anacardiaceae & & $0.01 \%$ & $0.26 \%$ & $0.11 \%$ \\
\hline Solanaceae & Solanum sessile Ruiz \& Pav. & & $0.20 \%$ & & $0.11 \%$ \\
\hline Betulaceae & Alnus sp & & $0.32 \%$ & & $0.10 \%$ \\
\hline Piperaceae & Piperaceae & & $0.16 \%$ & $0.01 \%$ & $0.10 \%$ \\
\hline Poaceae & Poaceae & & $0.12 \%$ & $0.05 \%$ & $0.09 \%$ \\
\hline Proteaceae & Grevillea sp & & $0.13 \%$ & $0.02 \%$ & $0.08 \%$ \\
\hline Asteraceae & Asplundianthus $s p$ & & $0.14 \%$ & & $0.08 \%$ \\
\hline Asteraceae & Asteraceae Tipo I & & $0.12 \%$ & & $0.07 \%$ \\
\hline Asteraceae & Asteraceae Tipo II & $0.09 \%$ & $0.08 \%$ & $0.03 \%$ & $0.06 \%$ \\
\hline Buddlejaceae & Buddleja americana L. & & $0.10 \%$ & & $0.06 \%$ \\
\hline Asteraceae & Ophryosporus sp & & $0.09 \%$ & & $0.05 \%$ \\
\hline Polygalaceae & Polygalaceae & & & $0.11 \%$ & $0.05 \%$ \\
\hline Desconocido & Desconocido III & & & $0.10 \%$ & $0.04 \%$ \\
\hline Desconocido & Desconocido II & & $0.06 \%$ & & $0.03 \%$ \\
\hline Piperaceae & Piper sp Tipo II & & $0.05 \%$ & & $0.03 \%$ \\
\hline Amarilydaceae & Amarilydaceae & & $0.04 \%$ & & $0.03 \%$ \\
\hline Polygalaceae & Rumex sp & & $0.04 \%$ & & $0.03 \%$ \\
\hline Asteraceae & Ambrosia sp & & $0.07 \%$ & & $0.02 \%$ \\
\hline Desconocido & Desconocido I & & $0.04 \%$ & & $0.02 \%$ \\
\hline Bignoniaceae & Bignoniaceae & $0.45 \%$ & & & $0.02 \%$ \\
\hline Arecaceae & Arecaceae Tipo II & & & $0.04 \%$ & $0.02 \%$ \\
\hline Asteraceae & Asteraceae Tipo III & & $0.03 \%$ & & $0.01 \%$ \\
\hline Fabaceae & Tephrosia vogelii Hook. f. & & $0.03 \%$ & & $0.01 \%$ \\
\hline Rutaceae & Tipo Citrus sp & & $0.03 \%$ & & $0.01 \%$ \\
\hline Myrtaceae & Myrtaceae & & $0.01 \%$ & $0.02 \%$ & $0.01 \%$ \\
\hline Fabaceae & Tipo Acacia sp & & $0.01 \%$ & & $0.01 \%$ \\
\hline Euphorbiaceae & Acalypha macrostachya Hook. f. & & & $0.01 \%$ & $0.01 \%$ \\
\hline
\end{tabular}


método propuesto por Hidalgo \& Bootello (1990), asignándoseles códigos de la guía universal de colores PANTONE. Los grupos de un mismo color fueron nuevamente pesados para realizar cálculos de frecuencia, acetolizándose una muestra por cada grupo de color. Los granos de polen fueron identificados por comparación con los tipos polínicos de referencia y con la bibliografía.

\section{Resultados.}

Se determinaron 47 taxa que fueron utilizadas por Apis mellifera como fuente de polen de las cuales, Dictyocaryum lamarckianum "basanco", Juglans neotropica "nogal” y Cyrtocymura scorpioides fueron los recursos poliníferos más importantes en el valle de Oxapampa en la temporada 2007 (Tabla 2). La palmera Dictyocaryum lamarckianum constituyó la especie que aportó la mayor cantidad de polen para Apis mellifera en todo el valle de Oxapampa, estuvo presente en las muestras de los distritos de Chontabamba y Oxapampa, alcanzando valores mayores en Chontabamba, donde se evidenció una mayor distribución de esta especie.

Tabla 3. Taxa encontradas en los diferentes grupos de color en las muestras de polen corbicular en el distrito de Huancabamba.

\begin{tabular}{llrr}
\hline \multirow{2}{*}{ Taxa } & \multicolumn{2}{c}{ Color } & \multirow{2}{*}{ Porcentaje } \\
\cline { 2 - 3 } & CR & GP & \\
\hline Croton perspeciosus Croizat & Marrón & $731 \mathrm{M}$ & $87.16 \%$ \\
Cecropia sp & Marrón & $139 \mathrm{M}$ & $7.18 \%$ \\
Solanaceae Tipo II & Amarillo & $1215 \mathrm{M}$ & $5.13 \%$ \\
Bignoniaceae & Amarillo & $7408 \mathrm{M}$ & $0.45 \%$ \\
Asteraceae Tipo II & Naranja & $144 \mathrm{M}$ & $0.09 \%$ \\
\hline GP: Código asignado de acuerdo a la Guía universal de color PANTONE. \\
CR: Color referencial.
\end{tabular}

El género Cecropia "tacona” se halló presente en los tres distritos muestreados; sin embargo obtuvo frecuencias relativas bajas (Tabla 2), lo cual difiere de los resultados obtenidos por Villanueva (2002) y Da Luz et al. (2007), quienes reportaron una dominancia de Cecropia en las cargas polínicas que acopió Apis mellifera en la Península de Yucatán en México y en la región de Morro Azul do Tinguá en Brasil, respectivamente. Esto indicaría que Cecropia spp. a pesar de estar muy disponible, en nuestra área de estudio, no representa una fuente importante de polen para Apis mellifera, debido a la existencia de otras especies que poseen mejor calidad de polen para su alimentación, confirmando su selectividad por el recurso (Dávila, 1981; Ramírez \& Montenegro, 2004).

En el distrito de Huancabamba se identificaron 5 taxa, de los cuales Croton perspeciosus "sangre de grado” presentó la mayor frecuencia (Tabla 3), esta especie ha sido catalogada como endémica para el Perú y se sostiene que sus poblaciones han sido reducidas por la actividad agrícola, en especial por el cultivo del café (León et al., 2006). El uso apícola del género Croton ha sido reportado por diversos autores dándole mayor importancia como un recurso melífero que polinífero (Da Luz et al., 2007; Barth, 2004). También se ha reportado al género Croton como una fuente pobre de polen (Girón, 1995; Hatsue, 2006). Los resultados hallados en la presente investigación contrastan con los estudios mencionados anteriormente; pues Croton perspeciosus se presenta como una gran alternativa para la producción de polen en el distrito de Huancabamba.

En las muestras de polen corbicular del distrito de Chontabamba se determinó la presencia de 41 especies de plantas que fueron utilizadas por

Tabla 4. Taxa encontradas en los diferentes grupos de color en las muestras de polen corbicular en el distrito de Chontabamba.

\begin{tabular}{llll}
\hline \multirow{2}{*}{ Taxa } & \multicolumn{2}{c}{ Color } & Porcentaje \\
\cline { 2 - 4 } & CR & GP & \\
\hline Dictyocaryum lamarckianum (Mart.) H. Wendl & Amarillo & $7406 \mathrm{M}$ & $37.27 \%$ \\
Juglans neotropica Diels. & Marrón & $146 \mathrm{M}-1395 \mathrm{M}$ & $28.51 \%$ \\
Cyrtocymura scorpioides (Lam.) H. Rob & Marrón-Naranja & $7512 \mathrm{M}-718 \mathrm{M}$ & $13.89 \%$ \\
Trixis divaricata (Kunth) Spreng. & Marrón & $483 \mathrm{M}$ & $6.22 \%$ \\
Desconocido IV & Amarillo-Marrón & $7401 \mathrm{M}-7510 \mathrm{M}$ & $5.83 \%$ \\
Cucurbita sp & Marrón & $1535 \mathrm{M}-160 \mathrm{M}$ & $0.89 \%$ \\
Solanaceae Tipo I & Amarillo -Marrón & $100 \mathrm{M}-131 \mathrm{M}$ & $0.86 \%$ \\
Pinus tecunumanii F. Schwerdtf. ex Eguiluz \& J.P.Perry & Amarillo & $127 \mathrm{M}$ & $0.72 \%$ \\
Zea mays L. & Naranja & $145 \mathrm{M}-160 \mathrm{M}$ & $0.64 \%$ \\
Acnistus arborescens (L.) Schltdl. & Amarillo & $141 \mathrm{M}$ & $0.62 \%$ \\
Passiflora ligularis Juss. & Naranja, Marrón & $144 \mathrm{M}-1395 \mathrm{M}$ & $0.55 \%$ \\
Sida rhomboidea Roxb. ex Fleming & Marrón & $160 \mathrm{M}$ & $0.53 \%$ \\
Menores al 0.05\% & & & $3.47 \%$ \\
\hline
\end{tabular}

GP: Código asignado de acuerdo a la Guía universal de color PANTONE.

CR: Color referencial. 
Apis mellifera como fuente de polen, de las cuales Dictyocaryum lamarckianum "basanco", Juglans neotropica "nogal” y Cyrtocymura scorpioides, fueron los que aportaron la mayor cantidad de polen a la colmena (Tabla 4)

Dictyocaryum lamarckianum, "basanco”, que representó el mayor aporte polínico para el distrito de Chontabamba; no ha sido reportado anteriormente como un recurso polínifero; para Borchsenius \& Moraes (2006) esta especie de palmera tiene pocos usos, aún cuando produce una gran cantidad de polen que es empleado por Apis mellifera para producir polen corbicular de color amarillo; registrándose así, un uso alternativo para esta especie, utilizada mayormente en la construcción de cercos (Kahn \& Moussa, 1994), y ocasionalmente extraída por su palmito (Paniagua, 2005). Estos usos, han dejado restringida a esta especie a pocas localidades del valle de Oxapampa.

Los resultados del presente estudio indican que la especie Juglans neotropica, una especie arbórea empleada en la reforestación de ciertas zonas, representa una importante fuente de polen para esta localidad, reportándose así un uso diferente del árbol del nogal, además, de su uso como recurso maderero, medicinal y para extracción de tintes (Brack, 1999).

En el distrito de Oxapampa fueron identificadas 26 taxa poliníferas, de las cuales Dictyocaryum lamarckianum, Vernonanthura patens, Baccharis latifolia, Zea mays y Juglans neotropica presentaron el mayor aporte polínico, esto al parecer es debido a la disponibilidad de estas especies en la zona de estudio, ya sea como flora arbustiva nativa, recurso de reforestación o agrícola en el caso del maíz (Tabla 5), en esta zona Dictyocaryum lamarckianum también aportó la mayor cantidad de polen a la colmena, y a pesar de que solo se observó su presencia en un solo punto de muestreo, la cantidad colectada por Apis mellifera fue suficiente para ser considerada como la especie que aportó la mayor cantidad de polen para esta localidad.

Las especies Baccharis latifolia, Cyrtocymura scorpioides y Vernonanthura patens también fueron recursos poliníferos importantes; esto refleja el trabajo de campo, ya que se observó una gran abundancia de estos arbustos nativos en las afueras de la ciudad y sobre todo en las laderas de los cerros; la especie Baccharis latifolia, puede colonizar con gran facilidad terrenos que fueron talados para zona ganadera; es considerada una especie apícola importante por los apicultores del Valle de Oxapampa y ha sido reportada en ocasiones como un gran recurso melífero (Bonino \& Paucarmayta, 2002), mas no como recurso polinífero. Durante la presente investigación se observó gran abundancia de esta especie, sin embargo durante las últimas colectas se apreció el incremento de la poda de esta planta para ganar mayor área disponible destinada a la ganadería.

La especie Zea mays “maíz" representó un importante aporte polínico para el distrito de Oxapampa, a pesar de que el uso de poáceas como fuente polinífera es muy poco frecuente, pues sus flores son muy pequeñas, cubiertas por brácteas con colores poco llamativos para los insectos visitantes, $\mathrm{y}$ por estar generalmente adaptadas a la polinización por el viento o por gravedad (Crane \& Walker, 1984); nuestros resultados demuestran que este grupo de plantas pueden ser utilizadas por Apis mellifera como recurso polinífero alternativo cuando existe baja disponibilidad de especies que aporten polen alrededor de los apiarios; coincidiendo esto con lo descrito por Girón (1995) quien menciona que este grupo de plantas contribuyen al sostenimiento de las colmenas en épocas de escases o en áreas donde no hay una flora polinífera dominante.

Por último, durante el trabajo de campo se

Tabla 5. Taxa encontradas en los diferentes grupos de color en las muestras de polen corbicular en el distrito de Oxapampa.

\begin{tabular}{|c|c|c|c|}
\hline \multirow{2}{*}{ Taxa } & \multicolumn{2}{|r|}{ Color } & \multirow{2}{*}{ Porcentaje } \\
\hline & $\mathrm{CR}$ & GP & \\
\hline Dictyocaryum lamarckianum(Mart.) H. Wendl & Amarillo & $7405 \mathrm{M}$ & $24.14 \%$ \\
\hline Vernonanthura patens (Kunth) H. Rob. & $\begin{array}{l}\text { Amarillo, Marrón, } \\
\text { Naranja }\end{array}$ & $\begin{array}{l}\text { 7408M-7510-146M-7511M-1535M- } \\
\text { 724M }\end{array}$ & $20.66 \%$ \\
\hline Baccharis latifolia(Ruiz \& Pav.) & Marrón, Verde & 139M-119M & $14.95 \%$ \\
\hline Zea mays L. & Amarillo & $7408 \mathrm{M}$ & $11.41 \%$ \\
\hline Juglans neotropica Diels & Marrón & 469M-146M-1395M & $10.65 \%$ \\
\hline Cyrtocymura scorpioides (Lam.) H. Rob & Marrón, Naranja & 718M-717M-1525M-1545M-725M & $6.10 \%$ \\
\hline Croton perpeciosus Croizat & Marrón & $724 \mathrm{M}-731 \mathrm{M}$ & $2.99 \%$ \\
\hline Munnozia hastifolia(Poepp.) H. Rob. \&Brettell & Naranja & $1525 \mathrm{M}$ & $2.07 \%$ \\
\hline Cucurbita sp & Naranja & $7414 \mathrm{M}$ & $1.76 \%$ \\
\hline Sida rhomboidea Roxb. ex Fleming & Marrón, Naranja & 7512M-7414M & $1.65 \%$ \\
\hline Piper tipo $I(*)$ & Marrón, Naranja & 469M-1385M & $1.65 \%$ \\
\hline Menores al 1\% & & & $1.97 \%$ \\
\hline
\end{tabular}

GP: Código asignado de acuerdo a la Guía universal de color PANTONE.

CR: Color referencial. 
observó la presencia de plantaciones de Coffea arabica L. "café”, así como de diferentes especies de la familia Melastomataceae; sin embargo, ninguna de estas taxa se encontraron presentes en las muestras de polen corbicular analizadas; por lo que no representan especies de importancia polinífera. Así mismo, en todo el Valle de Oxapampa al borde de los caminos se encontraron diferentes especies de Inga spp "pacae”, que eran visitadas por las abejas, siendo considerada como una especie importante por los apicultores como flora melífera del Valle; sin embargo esta especie sólo representó el $0.16 \%$ de aporte de polen, por lo que tampoco puede ser considerada como una fuente polinífera importante; en cambio, a decir de los apicultores de la zona, esta especie sí representaría un recurso importante como flora melífera del Valle de Oxapampa. La especie Pinus tecunumanii "pino" es utilizada ampliamente en la reforestación del Valle de Oxapampa; sin embargo presentó frecuencias relativas bajas, aún cuando se encuentran en grandes cantidades, lo cual indicaría que pese a estar muy disponible no es preferido como recurso polínifero; lo que se justifica, por el hecho de que el polen del pino posee un bajo contenido de proteínas (Stanley \& Linskens, 1974); a esto se suma la presencia de otras especies con una mejor calidad de polen para las abejas, como lo son Dictyocaryum lamarckianum, Juglans neotropica y Cyrtocymura scorpioides cuya preferencia por la abeja se evidencia en los resultados obtenidos en el presente estudio.

\section{Conclusiones.}

- Las especies Dictyocaryum lamarckianum y Juglans neotropica son los recursos poliníferos más importantes en el Valle de Oxapampa. La especie Croton perspeciosus es el recurso polinífero más importante en el distrito de Huancabamba y Dictyocaryum lamarckianum, lo es en los distritos de Chontabamba y Oxapampa.

- Cyrtocymura scorpioides, Vernonanthura patens y Baccharis latifolia constituyen recursos poliníferos secundarios.

- Las especies más representativas del Valle de Oxapampa como, Cecropia spp, Inga spp y Pinus tecunumanii, no representan recursos poliníferos importantes.

- Apis mellifera posee una selectividad en el uso de los recursos poliníferos.

\section{Agradecimientos.}

A los apicultores del Valle de Oxapampa, en especial al Sr. Fritz Frey Nano, al Sr. Augusto Bottger y al Sr. Pablo Ayala por la ayuda prestada. Al Ing. Rodolfo Vásquez y a la Blga. Rocio Rojas, del Herbario Selva Central (HOXA) por la identificación de las especies vegetales.

\section{Literatura citada.}

Baldi B., Grasso D., Chaves S. \& Fernández G. 2004. Caracterización bromatológica del polen apícola argentino. Ciencia, Docencia y T0ecnología No 29, Año $\mathrm{XV}$. Investigación Ciencias Exactas y Naturales Ingenierías y Tecnologías (145-181).

Barth M. 2004. Melissopalinologia no brasil: Uma revisão sobre análises palinológicas de mel, própolis e bolotas de pólen de abelhas. Sci. Agric. 61 (.3): 342-350.

Bonino R. \& Paucarmayta D. 2002. Evaluación del potencial melífero en la margen derecha del río Mapacho sector Challabamba - Accobamba. PROMANU. Cusco.

Borchsenius F. \& Moraes M. 2006. Diversidad y usos de palmeras andinas (Arecaceae). Botánica Económica de los Andes Centrales. Universidad Mayor de San Andrés, La Paz. : 412-433.

Brack A. 1999. Diccionario Enciclopédico de Plantas Útiles del Perú. Centro de Estudios Regionales Andinos "Bartolomé de Las Casas" (CBC).

Carpes T. 2008. Estudo das Caracteristicas Físico químicas e Biológicas do polen apícola de Apis mellifera L. da regiao sul do Brasil. Tesis para obtener el Título de Doctor en Tecnología de Alimentos. Universidad Federal do Paraná.

Contreras O. 2004. Relación entre el Contenido de Caroteno, Color y Características Botánicas del Polen Corbicular. Tesis para optar al grado de Licenciado en Ingeniería en Alimentos. Universidad Austral de Chile. Facultad de Ciencias Agrarias. Escuela de Ingeniería en Alimentos.

Crane E. \& Walker P. 1984. Pollination Directory for World Crops. International Bee research Association. British Library Cataloguing in publication Data Crane, Eva. London, UK.

Da Luz C., Thomé M. \& Barth O. 2007. Recursos tróficos de Apis mellifera L. (Hymenoptera, Apidae) na região de MorroAzul do Tinguá, Estado do Rio de Janeiro. Revista Brasil. Bot. 30 (1): 29-36.

Da Silva L., Sarmento T., Amorrin C., Costa M., Ribeiro Dos Santos F. \& Agra Da Silva R. 2006. Estudo quimico, analise palinologica e atividade antiradicalar do polen apícola (Apis mellifera). Sociedad Brasileira de Química.

Dávila M. 1981. Principios de Apicultura. Departamento de Sanidad Vegetal Universidad Nacional Agraria La Molina.

Erdtman G. 1966. Pollen Morphology and Plant Taxonomy. Hafner Publishing Company.

Faye P., Planchuelo A. \& Molinelli M. 2002. Relevamiento de la Flora Apícola e Identificación de Cargas de Polen en el sureste de la provincia de Córdoba, Argentina. Agriscientia, VOL. XIX : 19-30.

Faye P. \& Molinelli M. 2004. Cargas de Polen Provenientes del Sureste de la Provincia de Córdoba, Argentina. Espacio Apícola. 72.

Fegúndez G.A \& Caccavara M.A. 2003. Primeros registros de mieles Monoflorales y Cargas de Polen de soja (Glycine max L.) en Argentina. Boletín Apícola Trimestral. 24: 3-6.

Fegúndez G., Muñoz J. \& Reinoso P. 2006. Caracterización, Fenología e Interés Apícola de la Vegetación del Departamento Diamante, Entre Ríos. Espacio Apícola. 72: 32-33: 
Girón V. 1995. Análisis Palinológico de la Miel y la Carga de Polen Colectada por Apis mellifera en el Suroeste de Antioquia, Colombia. Bol. Mus. Ent. Universidad Valle. 3 (2): 35-54.

Hatsue A., Fernández C. \& Meira A. 2007. Composição e qualidade de pólen apícola coletado em Minas Gerais. Pesq. agropec. bras., Brasilia. 42. (8) :1057-1065.

Hatsue A. 2006. Flora e caracteriçao polinífera para abelhas Apis mellifera L. na regiao da Vicosa, M.G. Tesis para optar el grado de Magister Scientiae. Universidad Federal de Vicosa.

Hidalgo M.I. \& Botello M.L. 1990. Origen floral de las cargas de polen recogidas por Apis mellifera L. en Alora (Málaga). Acta Bot. Malacitana. 15: 33-44.

Isayama V. 1987 -1988. Morfología polínica de algunas especies de la flora del Dpto. de Lambayeque. Zonas Áridas. 5:45-60.

Kahn F. \& Moussa F. 1994. Las Palmeras del Perú. Instituto Francés de estudios andinos. IFEA.

Lazcano H., Navarro M., Ávila R., Dávila R. \& Marroquin R. 2006. Análisis Químico- Nutrimental y Palinológico de polen Recolectado por Abejas en las Regiones IV, V, VI del estado de Puebla. Facultad de Ciencias Químicas, Benemérita Universidad Autónoma de puebla.

León B., Riina R. \& Berry P. 2006. Euphorbiaceae Endémicas del Perú. Rev. peru. biol. Número especial 13(2): 295s - 301s.

León O., Balbín J., Villa V. \& Isayama V. 1989 - 1990. Origen botánico de la miel del Valle del Mantaro. Zonas Áridas. 6: 95-108.

Montenegro G., Gómez M. \& Ávila G. 1992. Importancia relativa de especies cuyo polen es utilizado por Apis mellifera en el área de la reserva nacional Los Ruiles, VII Región de Chile. Acta Botánica Malacitana. 17: 167-174.

Montenegro G. \& Pizarro R. 2006. Manejo de Apiarios, producción Apícola y certificación de Miel. Pontificia Universidad Católica de Chile Facultad de Agronomía e Ingeniería Forestal.

Paniagua N. 2005. Diversidad, densidad, distribución y uso de las palmas en la región de Madidi, noreste del departamento de La Paz (Bolivia). Ecología en Bolivia.
40 (3): 265-280.

Ramírez R. \& Montenegro G. 2004. Certificación del Origen Botánico de Miel y Polen Corbicular pertenecientes a la comuna de Litueche, VI Región de Chile. Ciencia e Investigación Agraria. 31(3): 197-211.

Sáenz C. \& Gómez C. 2000. Mieles Españolas Características e Identificación mediante el análisis del polen. Ediciones Mundi-Prensa Madrid.

Salamanca G., Guerra P.E., Vargas G.E. \& Henao R.C. 2002. Actividad del agua, condiciones microbiológicas y propiedades físico-químicas de las cargas de polen colectado y almacenados por Apis mellifera. XXXVII Congreso Nacional de Ciencias Biológicas (ACCB).

Sá-Otero M. P., Bugarín S.M., Armesto-Baztán S. \& DíazLosada E. 2002. Método de Determinación del Origen Geográfico del Polen Apícola Comercial. Lazaroa. 23: $25-34$.

Sempe J., Ramirez C. \& Montenegro G. 1989. Flora Utilizada como Fuente de Polen por Apis mellifera en la provincia de Valdivia: Análisis Cuantitativo de Polen Corbicular. Ciencia e Investigación Agraria. 16 (1-2).

Stanley R. \& Linskens H. 1974. Pollen, Biology Biochemistry Management. Springer-Verlag. New York.

Ventura K. \& Huamán L. 2008. Morfología polínica de la familia Fabaceae de la parte baja de los valles de Pativilca y Fortaleza (Lima- Perú). Biologist. 6: 112134.

Villanueva R. 2002. Polliniferous plants aud foraging strategles of Apis mellifera (Hymenoptera: Apidae) in the Yucatán Peninsula, Mexico. Rev. biol. Trop. 50 (34).

Zevallos P. 1986. Determinación de la flora apícola del Dpto. de Lambayeque. Fundación para el Desarrollo Nacional. Lima.

Zevallos P. \& Pérez E. 1990. Determinación del potencial melífero de los bosques secundarios de Pucallpa. Estudio Preliminar. UNALM. Fac. Ciencias Forestales. Centro Internacional de Investigaciones para el Desarrollo.

Zevallos P. \& Higaona R. 1988. Valor pecuario y apícola de 10 especies forestales de las zonas secas y semisecas de Lambayeque. Zonas Aridas. 5:31-43.

1 Laboratorio de Palinología y Paleobotánica, Sección de Ciencias Ambientales (LID), Facultad de Ciencias y Filosofía, Universidad Peruana Cayetano Heredia

2 Laboratorio de Palinología y Paleobotánica, Sección de Ciencias Ambientales (LID), Facultad de Ciencias y

Filosofía, Universidad Peruana Cayetano Heredia 